\title{
Effects in rats of adolescent exposure to Cannabis sativa on emotional behavior and adipose tissue
}

\author{
Kamali-Sarvestani A ${ }^{1}$, Hoseini SE ${ }^{1}$, Mehrabani D ${ }^{2,3,4}$, Hashemi $\mathrm{SS}^{3}$, Derakhshanfar $\mathrm{A}^{5}$ \\ Burn and Wound Healing Research Center, Shiraz University of Medical Sciences, Shiraz, Iran. \\ mehrabad@sums.ac.ir
}

\begin{abstract}
OBJECTIVE: Cannabis sativa L has remained the most widely used recreational and abused drug worldwide. This study determined adipose tissue histological changes and the anxiety-like behavior in elevated plusmaze tests in rats treated with $C$. sativa.

METHODS: C. sativa L. was provided from Islamic Azad University Herbarium. To reach cannabis powder, a rotary evaporator was used. Forty-five male Wistar rats were randomly divided into three equal groups of experimental receiving $2 \mathrm{mg} / \mathrm{kg}$ of cannabis extract dissolved in $70 \%$ ethanol in $0.6 \mathrm{~mL}$ volume subcutaneously daily for three weeks, the sham group subcutaneously injected with equal volume of $70 \%$ ethanol and the control receiving just distilled water, identically. To assess the anxiety level, elevated plus maze was used. Histopathological changes in adipose tissue was evaluated after 7, 14, and 21 days post-intervention.

RESULTS: After cannabis administration, inflammation, degeneration and necrosis in adipose tissue occured. Regarding the anxiety level for the percentage of time spent in open arm (OAT), the percentage of time spent in closed arm (CAT), the percentage of time spent in central parts, and head dipping over the side of the maze) after one week, no significant difference was noticed between the groups; but 2 and 3 weeks of cannabis use, the anxiety level significantly increased.

CONCLUSION: Histological findings denoted to inflammation, degeneration and necrosis in adipose tissue after cannabis use. Behavioral assessment of anxiety level revealed that one week after cannabis, no changes were seen in anxiety, but 2 and 3 weeks after cannabis use, the anxiety level increased significantly (Fig. 5, Ref. 38). Text in PDF www.elis.sk.

KEY WORDS: behavior, histology, adipose tissue, fat, cannabis sativa.
\end{abstract}

\section{Introduction}

Cannabis is a hemp plant in the genu flowering plants having three common species of sativa, indica, and ruderalis. The term Cannabis sativa from family Cannabaceae has common names like marijuana, weed, and grass and generally denotes to the dried flowers and subtending leaves and stems of the female cannabis plant as the most widely used medicinal plant in the world that dates back 5,000 years and is now the most consumed illicit drug worldwide $(1,2)$. It is a complex plant with over 400 chemical

${ }^{1}$ Department of Biology, Shiraz Branch, Islamic Azad University, Shiraz, Iran, ${ }^{2}$ Burn and Wound Healing Research Center, Shiraz University of Medical Sciences, Shiraz, Iran, ${ }^{3}$ Stem Cell Technology Research Center, Shiraz University of Medical Sciences, Shiraz, Iran, ${ }^{4}$ Center of Comparative and Experimental Medicine, Shiraz University of Medical Sciences, Shiraz, Iran, and ${ }^{5}$ Diagnostic Laboratory Sciences and Technology Research Center, School of Paramedical Sciences, Shiraz University of Medical Sciences, Shiraz, Iran

Address for correspondence: D. Mehrabani, Burn and Wound Healing Research Center, Shiraz University of Medical Sciences, Shiraz, Iran. Phone: +98.71.32341025

Acknowledgement: The authors would like to thank Shiraz Islamic Azad University for financial support and Comparative and Experimental Medicine Center of Shiraz University of Medical Sciences providing the laboratory facility for their kind cooperation. entities and its primary constituent responsible for most of the pharmacological effects is $\Delta 9$-tetrahydrocannabinol (THC) that was known almost 50 years ago (3).

There are other constituents including cannabidiol (CBD), cannabinol (CBN), cannabichromene (CBC) and cannabigerol (CBG), while THC and CBD are the best characterized of the cannabinoids (4). THC exerts its effect via activation of two types of cannabinoid receptors, called $\mathrm{CB} 1$ and $\mathrm{CB} 2$. The $\mathrm{CB} 1$ receptor is seen and expressed primarily in the brain and then in peripheral tissues, but the $\mathrm{CB} 2$ receptor is visible and expressed primarily in peripheral tissues, and then in neuroglial cells (5).

Cannabis has been widely used for recreational purposes with over 190 million users worldwide, while over $20 \%$ of the population were reported as regular users (6). In US, there are almost 11 million users between 18 and 25 years due to decriminalization or legalization of cannabis in many states of the country (7). Based on 2018 census, a total of 31 states in the United States have issued laws to permit access for medical cannabis (8). Approximately, half of the 12 years or older US population have reported use of cannabis at least once during their lifetime (7).

Among 50 years or older individuals from 2006 to 2013, the rate of last year cannabis use was demonstrated to be around $71.4 \%$ (9), while half of them were infrequent users, taking cannabis once a month or less (10). Cannabis interferes with the body's chemical 
balance and affects a number of systems and organs with behavioral and physiological effects. Individuals become dependent on it at a young age and its high lipid-solubility leads to the persistence in the body for long periods of time (11).

Cannabis is available in different forms such as dried leaves, seeds, flowers, or oil and also the route of administration is different including inhalation as the most common method of use undertaken via smoking or vaporizing, and oral consumption is the other route. Inhalation is the fastest method of intoxication and with more bioavailability, while the THC plasma level reaches the maximum peak after 15-30 min. Oral consumption has a slower effect, usually reaching the peak level at $1-3 \mathrm{~h}$, and lasts $4-12 \mathrm{~h}$. The bioavailability of oral consumption is less than inhalation, due to difficulties to control the amount of active ingredients in the formulations and individual variations in absorption from the gut (12).

Worldwide, the cannabis usage has increased both for medicinal and recreational purposes, so understanding of plant cannabinoid effects seem necessary. The hyperphagic effect of THC in humans and rodents was previously shown (13). Several reports described poisoning by oral cannabis in children with the most common neurologic symptoms including lethargy, ataxia, tachycardia, mydriasis, respiratory depression, and hypotonia (14). The effect of THC on energy regulation has been conducted with conflicting results. It was shown that the endocannabinoid system mediates feeding behavior and energy balance, because THC exerts a biphasic dose response, with appetite stimulation at low doses and inhibition at high doses and identically on glucose uptake stimulating uptake at low THC levels, and inhibiting at high THC levels (15).

It was shown that cannabis extract protects pancreatic islets against the negative effects of obesity (15). Cannabinoid receptors (CB1R and CB2R) and their endogenous ligands, the endocannabinoids, have an important role in energy balance regulation, appetite, insulin sensitivity, pancreatic b-cell function, and lipid metabolism (16). Consequently, this study was designed to determine adipose tissue histological changes and the anxiety-like behavior in elevated plus-maze tests in rats treated with $C$. sativa.

\section{Material and methods}

\section{Preparation of C. sativa L. extract}

C. sativa L. was provided from Islamic Azad University Herbarium, Shiraz Branch, Shiraz, Iran. The whole plant was left at room temperature in shadow condition to be dried and then was changed to powder, while the particle sizes were not greater than $4.5 \mathrm{~cm}$. Maceration method was applied for extraction. The powder was kept in a tightly closed container containing $70 \%$ ethanol for 2-4 days. Meanwhile, it was shaken once or twice per day. The resulting solution was then filtered and the solute was isolated using a rotary evaporator. After that, the extract was left in oven at $40^{\circ} \mathrm{C}$ to be dried. The dried extract was finally dissolved in $70 \%$ ethanol to reach the requested concentrations for further experiments.

\section{Animals}

Forty-five male 8 weeks 200-220 g Wistar rats were purchased from Comparative and Experimental Medicine Center of Shiraz University of Medical Sciences, Shiraz, Iran. The rats were allowed to acclimatize to their condition kept at $22 \pm 1{ }^{\circ} \mathrm{C}$ with $12 \mathrm{~h}$ light/dark cycle, and had free access to food and water. Animals were fed with a standard rodent diet. The Animal Care Committee of Shiraz Islamic Azad University Ethics Committee (7-E-IR-MIAU.REC.80-B-1397) approved all experiments, and all procedures were conducted in accordance with the guidelines of working with laboratory animals of Iran Veterinary Organization. The rats were randomly divided into three equal groups. Experimental group received subcutaneous injection of $2 \mathrm{mg} /$ $\mathrm{kg}$ of the extract dissolved in $70 \%$ ethanol in 0.6 volume daily for three weeks, sham group just received equal volume of $70 \%$ ethanol similarly and the control group received distilled water, identically. Animals received cannabis daily between 08:00 AM and 12:00 AM. Rats were then euthanized by decapitation under ether anesthesia for tissue collection.

\section{Histopathological assessment}

Adipose tissue was provided after animals were euthanized and were fixed in $10 \%$ buffered formaldehyde. The tissues were dehydrated in two changes of cold ethanol, cleared by passing through three changes of cold xylene, and embedded in paraffin at $53{ }^{\circ} \mathrm{C}$. Serial sections were cut and slides were dried for 1 hour at $37^{\circ} \mathrm{C}$ and then stained using haematoxylin and eosin, while 5-mm thick sections were used and evaluated under light microscope in time intervals of 7, 14, and 21 days after the administration.

\section{The elevated plus-maze test of anxiety}

The plus-maze had two opposite open arms of $50 \times 10 \mathrm{~cm}$, and two opposite arms that were surrounded by $40 \mathrm{~cm}$ high walls. The arms were connected by a central $10 \times 10 \mathrm{~cm}$ square to create a "plus" shape that was elevated $50 \mathrm{~cm}$ from the floor and was lit by the dim light. All experiments were video-recorded using a camera that was positioned above and at approximately $50^{\circ}$ to the maze for scoring the anxiety in a neighborhood room. The entries were counted together with the percent time spent in open arm (OAT), closed arm (CAT), and central parts of the maze. Head dipping evaluated the exploratory movement of head/shoulders over the side of the maze. OAT presented a negative correlation with anxiety but CAT illustrated a positive correlation with anxiety (17).

The arm entrance was considered when the rat's 4 paws entered the arm; and exit from the arm was defined as leaving of the forepaws from the arm. On the test day, the animals were transferred to the dimly illuminated laboratory and left undisturbed for at least $1 \mathrm{~h}$ before starting the experiments. A cylindrical cardboard tube was used for individual transportation of the rats from their cage to the plus-maze, rats were reactive to direct human handling. Testing was performed between 8.00 AM and 12.00 AM. An observer who was blinded to the intervention scored it for 5 min and directly collected the data in a computer. The maze was thoroughly wet and dry cleaned between the successive sessions.

\section{Statistical analysis}

The obtained data were presented as mean \pm SEM and analyzed with SPSS software (Version 20, Chicago, IL, USA) using 

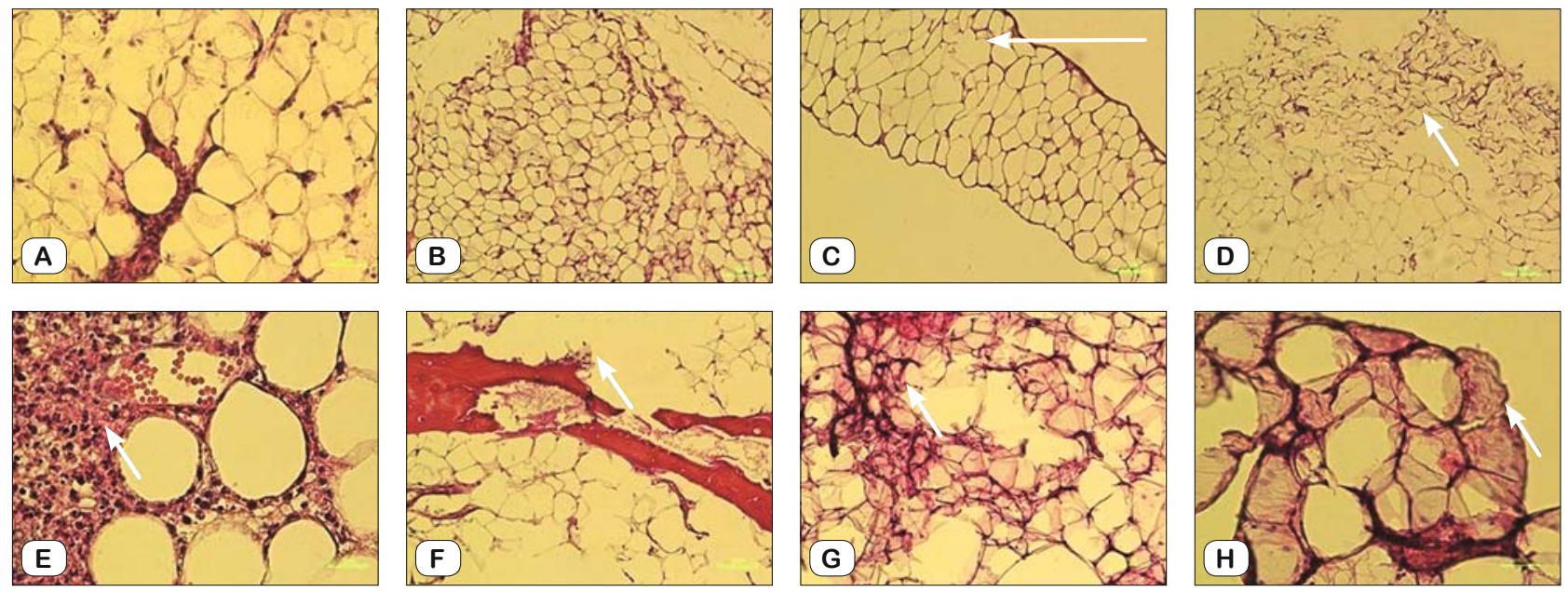

Fig. 1. A photomicrograph of a paraffin section of the adipose tissue after administration of cannabis in different groups of rats. It shows A: Control, Fat, normal (X200, H\&E); B: Sham, 1st week, adipose tissue, normal (X100, H\&E); C: Sham, 2nd week, adipose tissue, normal (X100, H\&E); D: Sham, 3rd week, adipose tissue, collapse marked by arrow (X100, H\&E); E: Cannabis, 1st week, adipose tissue, mononuclear cells inflammation, steatitis, increase in connective tissue marked by arrow (X100, H\&E); F: Cannabis, 2nd week, adipose tissue, exudate secretion, lobulation marked by arrow (X100, H\&E); G: Cannabis, 3rd week, adipose tissue, saponification, degeneration, necrosis marked by arrow (X100, H\&E); H: Cannabis, 3rd week, adipose tissue, saponification, degeneration, necrosis marked by arrow (X200, H\&E).

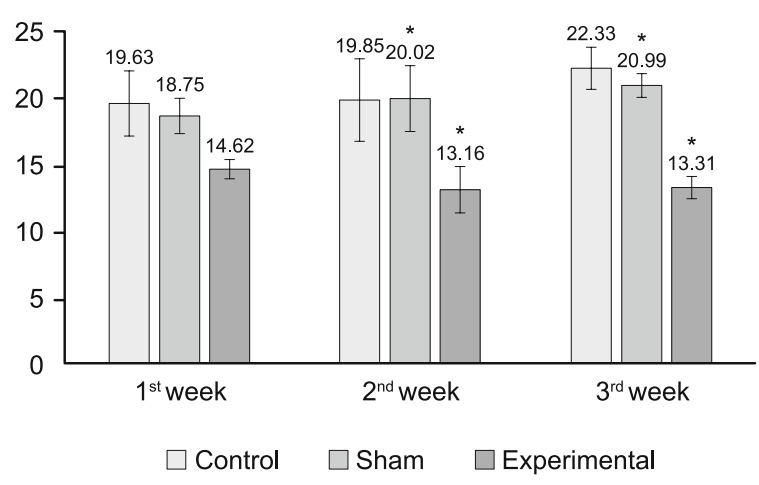

Fig. 2. The effect of cannabis on the mean percentage of time in open arm time (OAT) at the end of the first, second and third week between the control, sham and the experimental group showing no significant difference $(* \mathbf{p}<0.001)$.

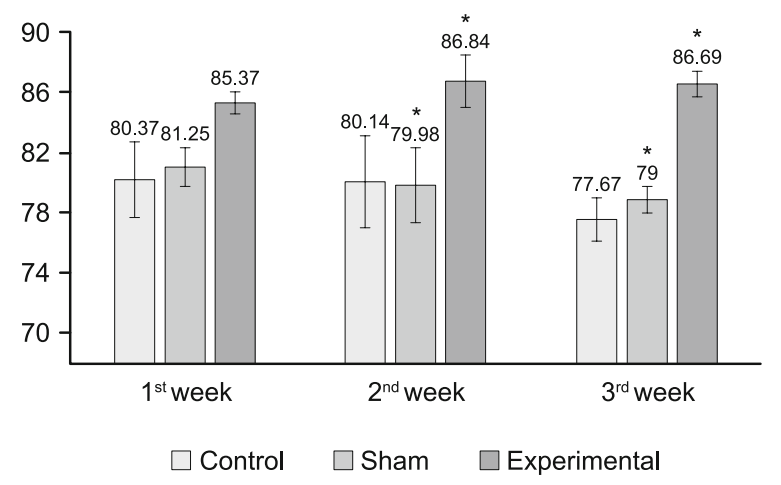

Fig. 3. The effect of cannabis on the mean percentages of time in closed arm time (CAT) at the end of the 1st, 2nd, and 3rd week between the control, the sham and the experimental group $\left({ }^{*} \mathbf{p}<0.001\right)$.

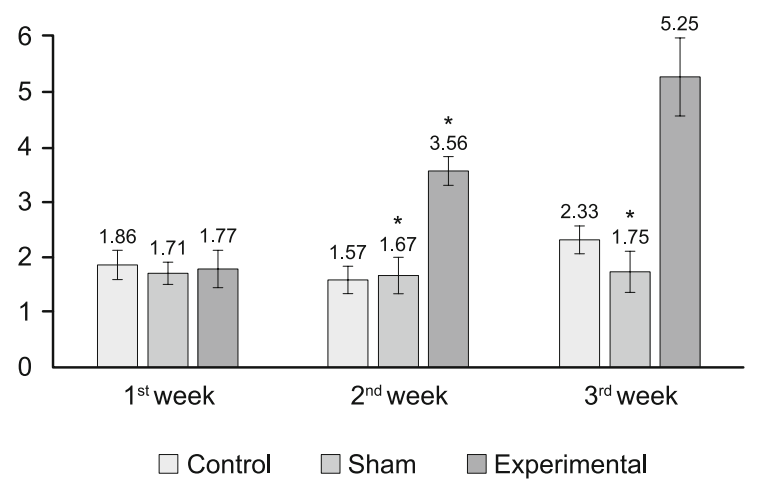

Fig. 4. The effect of cannabis on the mean number of times rats passing from the central parts of the maze at the end of the first, second and third week between the control, the sham and the experimental group $\left({ }^{*} \mathbf{p}<0.001\right)$.

the Independent $t$, One-Way ANOVA and Tukey tests to compare the groups. A p value less than 0.05 was considered statistically significant.

Results

\section{Histopathological assessment}

Pathological findings in adipose tissue after in vivo administration of cannabis in different groups of rats were shown in Figure 1 demonstrating that a week after cannabis use, inflammation and infiltration of mononuclear cells and an increase in connective tissue happened. After two weeks, exudate secretion and lobulation in adipose tissue were prominent and after three weeks; saponification, degeneration, and necrosis were visible. 


\section{7-301}

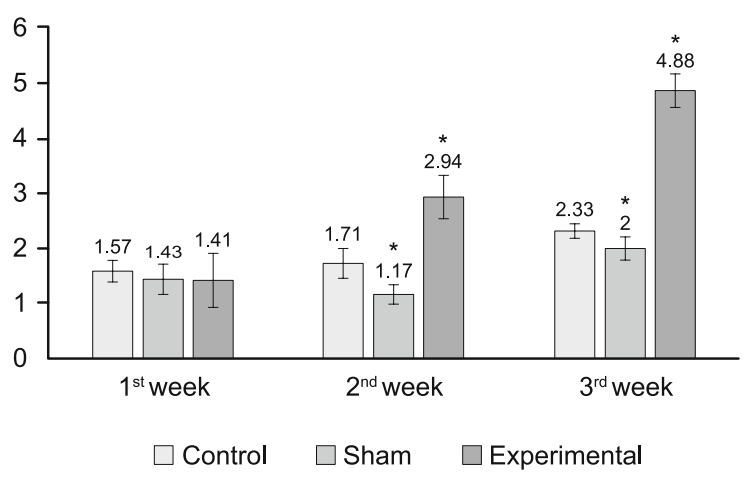

Fig. 5. The effect of cannabis on the mean number of head dipping at the end of the first, second and third week between the control, the sham and the experimental group $\left({ }^{*} \mathrm{p}<0.001\right)$.

\section{The elevated plus-maze test of anxiety}

One week after cannabis use, OAT, CAT, percent time spent in central parts of the maze and head dipping of the animals did not show any significant difference between all groups that denotes to the absence of any anxiety following cannabis consumption (Figs 2-5). After 2 and 3 weeks of cannabis administration, the difference between the sham and experimental group regarding the OAT, CAT, percentage of time spent in central parts of the maze and head dipping of the animals was significant, revealing a significant increase in anxiety level 2 and 3 weeks following cannabis use $(\mathrm{p}<0.001)$ (Figs 2-5).

\section{Discussion}

Cannabis comes from the Cannabaceae with three major species of C. sativa, C. indica, and C. ruderalis that possess various potencies in two major active ingredients of delta-9-tetrahydrocannabinol (THC) and cannabidiol (CBD). C. sativa contains the highest level of THC and a low level of CBD (18). There has been an increase in cannabis use and cannabis-related emergency department visits (19). The rate of use has increased due to trends towards a more permissive legal environment (20). THC is responsible for the euphoric and psychoactive effect of cannabis (21).

Cannabis may have a negative impact on the cardiovascular system (22). The first report of cannabis allergy dates back to 1971 significantly affected the quality of life (23). In kidney transplant candidates, cannabis abuse was associated with psychiatric comorbidities (24). Cannabis can cause behavioral changes, and movement disturbances, while higher doses inhibit movement and lower doses stimulate it (24). In our study, similarly, behavioral assessment revealed an increasing trend for anxiety level after 2 and 3 weeks following cannabis use.

Cannabis use can cause cellular damage through mediation of imbalance and altered cytoarchitecture and results in changes of plasma ALP, ALT, AST levels (25). Iritani et al observed low feeding during a 14-day intraperitoneal cannabis treatment (26). Mazidi et al demonstrated that $\mathrm{C}$. sativa had a dose-related positive effect on appetite in experimental rats (27). THC was found to exert a biphasic dose response, with appetite stimulation at low doses and inhibition at high doses (28). Intravenous THC administration was shown to exert a dose-dependent dual effect on glucose uptake, stimulating uptake at low THC levels $(0.2 \mathrm{mg} / \mathrm{kg})$, and inhibiting uptake at high THC levels (5-10 mg/kg) (15).

Its effect on energy regulation has been variable with conflicting results. Cota et al reported a lag in weight gain following cannabis use as endocannabinoid system mediated feeding behavior and energy balance (29). The protective effect of cannabis on pancreatic islets against the negative effects of obesity was previously shown (15). In obese cannabis treated rats, an impaired weight gain was noted that may be due to an increased energy metabolism (30). In regulation of energy balance, appetite, insulin sensitivity, pancreatic b-cell function, and lipid metabolism, cannabinoid receptors (CB1R and CB2R) and their endogenous ligands, the endocannabinoids were shown to play important roles (16).

THC by affecting CB1 receptors, but not CB2 receptors, can stimulate glycolysis and activation of intracellular G-proteins leading to signals to several effectors such as ion channels, the mitogen-activated protein kinase (MAPK) cascade and induce myelocytomatosis oncogene (c-MYC) expression (31). Endocannabinoids (anandamide and 2-arachidonoyl glycerol) and CB1 receptors are seen in peripheral tissues involved in energy homeostasis, such as adipose tissue, pancreas, skeletal muscle, and liver (16). Our histological findings confirmed these effects denoted to degeneration, and necrosis in adipose tissue after cannabis use.

$\mathrm{CB} 1$ receptor activation leads to lipogenesis in the adipose tissue and liver (16), decreases insulin response in skeletal muscle (32), and impairs insulin activity and secretion in pancreatic b-cells (33). It was demonstrated that $\mathrm{CB} 1$ receptor antagonism promotes a decrease in body weight, an improvement in dyslipidemia, and attenuates insulin resistance (34). It was found that chronic cannabis usage was associated with visceral adiposity and adipose tissue insulin resistance (35).

Our results demonstrated a significant increase in anxiety level 2 and 3 weeks after cannabis use. Fear and anxiety were mentioned by many researchers with possible explanations for how trait anxiety is different from trait fear $(36,37)$. In healthy population, anxiety can facilitate efforts to maintain safety and well-being (38). Nevertheless, when these, often adaptive states, are prominent, it can turn physically and psychologically (e.g., agoraphobia) harmful (36).

\section{Conclusion}

Histological findings denoted to inflammation, degeneration, and necrosis in adipose tissue after cannabis use. Behavioral assessment on anxiety level revealed that one week after cannabis use, anxiety did not change, but after 2 and 3 weeks, the anxiety level demonstrated a significant increasing trend.

\section{References}

1. Jamshidi M, Hosseini SE, Mehrabani D, Amini M. Effect of hydroalcoholic extract of cannabis sativa on cell survival and differentiation of mesenchymal stem cells derived from human adipose tissue to osteoblast-like cells. J Gorgan Univ Med Sci 2019; 21: 50-58. 
2. Jamshidi M, Hosseini SE, Mehrabani D, Amini M. Effect of hydroalcoholic extract of cannabis (cannabis sativa) on morphology and the process of human adipose-derived mesenchymal stem cell growth. Electron J Gen Med 2018; 15: em31.

3. Gaoni Y, Mechoulam R. Isolation, structure, and partial synthesis of an active constituent of hashish. J Am Chem Soc 1964; 86: 1646-1647.

4. El-Alfy AT, Ivey K, Robinson K, Ahmed S, Radwan M, Slade D, Khan I et al. Antidepressant-like effect of $\Delta 9$-tetrahydrocannabinol and other cannabinoids isolated from Cannabis sativa L. Pharmacol Biochem Behav 2010; 95: 434-442.

5. Lopez-Rodriguez AB, Siopi E, Finn DP, Marchand-Leroux C, GarciaSegura LM, Jafarian-Tehrani M et al. CB1 and CB2 cannabinoid receptor antagonists prevent minocycline-induced neuroprotection following traumatic brain injury in mice. Cereb Cortex 2015; 25: 35-45.

6. Degenhardt L, Whiteford HA, Ferrari AJ, Baxter AJ, Charlson FJ, Hall WD et al. Global burden of disease attributable to illicit drug use and dependence: findings from the Global Burden of Disease Study 2010. Lancet 2013 ; 382: 1564-1574.

7. Substance Abuse and Mental Health Services Administration. Behavioral Health Statistics and Quality. Rockville, Maryland 20857, September 7, 2018.

8. National Conference of State Legislatures. State Medical Marijuana Laws. www.ncsl.org/research/health/state-medicalmarijuana-laws.aspx 2016.

9. Han BH, Sherman S, Mauro PM, Martins SS, Rotenberg J, Palamar JJ. Demographic trends among older cannabis users in the United States, 2006-13. Addiction 2017; 112: 516-525.

10. Blazer DG, Wu L. Nonprescription use of pain relievers by middleaged and elderly community-living adults: National Survey on Drug Use and Health. J Am Geriatr Soc 2009; 57: 1252-1257.

11. Iversen L. Cannabis and the brain. Brain 2003; 126: 1252-1270.

12. Grotenhermen F. Pharmacokinetics and pharmacodynamics of cannabinoids. Clin Pharmacokinet 2003; 42: 327-360.

13. Farrimond JA, Hill AJ, Whalley BJ, Williams CM. Cannabis constituents modulate $\delta 9$-tetrahydrocannabinol-induced hyperphagia in rats. Psychopharmacology (Berl) 2010; 210: 97-106.

14. Claudet I, Le Breton M, Brehin C, Franchitto N. A 10-year review of cannabis exposure in children under 3 years of age: do we need a more global approach? Eur J Pediatr 2017; 176: 553-556.

15. Levendal R A, Schumann D, Donath M, Frost CL. Cannabis exposure associated with weight reduction and $\beta$-cell protection in an obese rat model. Phytomedicine 2012; 19: 575-582.

16. Pacher P, Batkai S, Kunos G. The endocannabinoid system as an emerging target of pharmacotherapy. Pharmacol Rev 2006; 58: 389-462.

17. Holmes A, Parmigiani S, Ferrari PF, Palanza P, Rodgers RJ. Behavioral profile of wild mice in the elevated plus-maze test for anxiety. Physiol Behav 2000; 71: 509-516.

18. Singh A, Saluja S, Kumar A, Agrawal S, Thind M, Nanda S et al. Cardiovascular complications of marijuana and related substances: a review. Cardiol Ther 2018; 7: 45-59.

19. Substance Abuse and Mental Health Administration. Results from the 2016 National Survey on Drug Use and Health: Detailed Tables. Rockville: Center for Behavioral Health Statistics and Quality; 2017. https:// www.samhsa.gov/data/sites/default/files/NSDUHDetTabs-2016/NSDUHDetTabs-2016.pdf.

20. Hasin DS. US epidemiology of cannabis use and associated problems. Neuropsychopharmacology 2018; 43: 195-212.
21. Badowski ME, Perez SE. Clinical utility of dronabinol in the treatment of weight loss associated with HIV and AIDS. HIV AIDS (Auckl) 2016; 8: 37-45.

22. Ghosh M, Naderi S. Cannabis and Cardiovascular Disease. Curr Atheroscler Rep 2019; 21: 21.

23. Decuyper II, Rihs HP, Van Gasse AL, Elst J, De Puysseleyr L, Faber MA et al. Cannabis allergy: what the clinician needs to know in 2019. Expert Rev Clin Immunol 2019; 15: 599-606.

24. Stark AL, Hickson LJ, Larrabee BR, Thusius NJ, Karpyak VM, Hall-Flavin DK et al. Cannabis abuse and dependence in kidney transplant candidates. J Psychosom Res 2019 Apr 12. pii: S0022-3999(18)31010-9.

25. Abey NO. Cannabis sativa (Marijuana) alters blood chemistry and the cytoarchitecture of some organs in Sprague Dawley rat models. Food Chem Toxicol 2018; 116: 292-297.

26. Iritani S, Tohgi M, Arai T, Ikeda K. Immunohistochemical study of theserotonergic neuronal system in an animal model of the mood disorder. Exp Neurol 2006; 201: 60-65.

27. Mazidi M, Baghban Taraghdari S, Rezaee P, Kamgar M, Jomezadeh MR, Akbarieh Hasani $\mathbf{O}$ et al. The effect of hydroalcoholic extract of Cannabis Sativa on appetite hormone in rat. J Complement Integr Med 2014; 11: 253-257.

28. Megulies JE, Hammer RP. 9-Tetrahydrocannabinol alters cerebral metabolism in a biphasic. Dose-dependent manner in rat brain. Eur J Pharmacol 1991; 202: 373-378.

29. Cota D, Marsicano G, Lutz B, Vicennati V, Stalla GK, Pasquali R et al. Review: endogenous cannabinoid system as a modulator of food intake. Int J Obes Metab Disord 2003; 27: 289-301.

30. Wallenius K, Wallenius V, Sunter D, Dickson SL, Jansson JO. Intracerebroventricular interleukin-6 treatment decreases body fat in rats. Biochem Biophys Res Commun 2002; 293: 560-565.

31. Howlett AC, Barth F, Bonner TI, Cabral G, Casellas P, Devane WA et al. International union of pharmacology. XXVII. Classification of cannabinoid receptors. Pharmacol Rev 2002; 54: 161-202.

32. Liu J, Zhou L, Xiong K, Godlewski G, Mukhopadhyay B, Tam J et al. Hepatic cannabinoid receptor-1 mediates dietinduced insulin resistance via inhibition of insulin signaling and clearance in mice. Gastroenterology 2012; 142: 1218-1228.

33. Kim W, Lao Q, Shin Y K, Carlson OD, Lee EK, Gorospe M et al. Cannabinoids induce pancreatic $\beta$-cell death by directly inhibiting insulin receptor activation. Sci Signal 2012; 5: ra23.

34. Triay J, Mundi M, Klein S, Toledo F G, Smith SR, Abu-Lebdeh H et al. Does rimonabant independently affect free fatty acid and glucose metabolism? J Clin Endocrinol Metab 2012; 97: 819-827.

35. Muniyappa R, Sable S, Ouwerkerk R, Mari A, Gharib AM, Walter $\mathbf{M}$ et al. Metabolic effects of chronic cannabis smoking. Diabetes Care 2013; 36: 2415-2422.

36. Sylvers P, Lilienfeld SO, LaPrairie $\mathbf{J}$ L. Differences between trait fear and trait anxiety: implications for psychopathology. Clin Psychol Rev 2011; 31: 122-137.

37. Drayer RA, Piraino B, Reynolds CF 3rd, Houck PR, Mazumdar S, Bernardini $\mathbf{J}$ et al. Characteristics of depression in hemodialysis patients: symptoms, quality of life and mortality risk. Gen Hosp Psychiatry 2006; 28: 306-312.

38. Lang PJ, Davis M, Ohman A. Fear and anxiety: Animal models and human cognitive psychophysiology. J Affect Disord 2000; 61: 137-159.

Received September 22, 2019. Accepted January 9, 2020. 